\title{
LOCAL REFINEMENT TECHNIQUES FOR ELLIPTIC PROBLEMS ON CELL-CENTERED GRIDS I. ERROR ANALYSIS
}

\author{
R. E. EWING, R. D. LAZAROV, AND P. S. VASSILEVSKI
}

\begin{abstract}
A finite difference technique on rectangular cell-centered grids with local refinement is proposed in order to derive discretizations of second-order elliptic equations of divergence type approximating the so-called balance equation. Error estimates in a discrete $H^{1}$-norm are derived of order $h^{1 / 2}$ for a simple symmetric scheme, and of order $h^{3 / 2}$ for both a nonsymmetric and a more accurate symmetric one, provided that the solution belongs to $H^{1+\alpha}$ for $\alpha>\frac{1}{2}$ and $\alpha>\frac{3}{2}$, respectively.
\end{abstract}

\section{INTRODUCTION}

The need for local refinement in solving real-life problems is of great practical importance even for today's computer facilities. One can substantially save computer storage and time in exploring the local properties of the solution of a differential problem that describes, for example, a certain physical phenomenon, if the discretization method takes advantage of these local properties of the solution. Often, in practice, local properties of the solution are not known in advance but can be determined by certain a posteriori analysis.

In this paper, we consider the problem of how to construct conservative approximations of divergence-type second-order elliptic boundary value problems on grids with local refinement. Local grid refinement for such types of problems has been used widely in the petroleum engineering literature, e.g., $[7,13,14$, 20]. The importance of very careful treatment of the difference stars near the composite grid interfaces has been demonstrated experimentally in [14]. This problem is the main concern of the present paper.

Cell-centered approximations were first proposed, studied, and applied to certain 1-D parabolic problems by Samarskii in [15]. From a mixed finite element approximation of 2-D second-order elliptic problems with mass lumping that allows elimination of the velocity, Weiser and Wheeler [21] obtain and analyze five-point cell-centered schemes on nonuniform but rectangular grids.

Received April 24, 1989; revised November 13, 1989.

1980 Mathematics Subject Classification (1985 Revision). Primary 65N05, 65N15.

Key words and phrases. Cell-centered grid, local refinement, error estimates, elliptic problems of divergence type. 
The coefficients of these schemes are harmonic mean values of the coefficients of the differential equation. As discussed in detail in [4], this is an important feature of the schemes for problems with piecewise smooth coefficients, if mass conservation is desired.

In this paper, we adopt the strategy of regular local grid refinement (see, e.g., [5]) when each grid cell in a certain local subdomain is subdivided into a number of rectangular cells. Then the discretization method used is based on the finite volume technique by approximation of the balance equation over each grid cell (see $[9,15,16,21])$. As a consequence, finite difference schemes derived in this manner on the composite grid preserve mass balance exactly. With the exception of the irregular cells adjacent to the interfaces of the refined subregion and the remaining part of the region, this approximation leads to the standard 5-point difference equation (see, e.g., $[16,19,21]$ ). We treat the irregular grid cells very carefully, proposing three new approximations of the balance equation. Thus, our local refinement technique can be considered as an extension of the results from Weiser and Wheeler [21] to the case of grids with regular local refinement.

The error analysis of the derived difference schemes is done in the framework of the book by Samarskii, Lazarov, and Makarov [18]. Since we are approximating the balance equation, the local truncation error can be presented in a divergence form with components depending only on the first derivatives of the exact solution. Then, using the Bramble-Hilbert lemma argument [6], we provide an estimate of these residuals that require $H^{m}, m>\frac{3}{2}$, regularity of the solution. In this way, we prove an $O\left(h^{\alpha}\right)$ convergence rate for the difference schemes in a discrete $H^{1}$-norm, with $\alpha=\frac{1}{2}$ for the simplest symmetric scheme, and $\alpha=\frac{3}{2} \quad(m>1+\alpha)$ for the nonsymmetric and a more accurate symmetric scheme.

Unlike the finite element method, the finite difference approximation of a selfadjoint elliptic problem can produce a linear algebraic problem with a nonsymmetric matrix. In many cases, this is a severe violation of the fundamental physical principle of reciprocity and should be avoided. There is also a purely mathematical reason for avoiding nonsymmetry. Many efficient methods for solving large systems of linear algebraic equations (for example, the conjugate gradient method) rely on the symmetry of the matrix. In order to apply these methods in a most efficient manner, we have to construct symmetric difference schemes. This problem is also extensively studied in the present paper, where we derive two symmetric approximations and study their algebraic and convergence properties. However, in the case of local refinement, the most natural approximation near the composite grid interfaces produces a nonsymmetric scheme. It appears that this nonsymmetry is not too severe. We study the algebraic properties of the corresponding matrix in order to use a generalized conjugate gradient, e.g., that from Axelsson [2], in a subsequent paper.

The remainder of the paper is organized as follows. In $\S 2$, we introduce the necessary notation, formulate the problem, and state our principles for the finite 
difference approximation. On the basis of the balance equation, we derive the finite difference approximations in $\S 3$. Special attention is paid to the approximation at the irregular grid points near the interface between the coarse and fine grids, and three different approximations are derived. Then we formulate the discrete problem as a system of linear algebraic equations and prove that the corresponding matrix has a positive definite symmetric part. Next, we study in $\S 4$ the discrete problem for the error of the method, presenting the local truncation error in a special divergence form. This enables us to prove convergence of the schemes with a rate of $O\left(h^{\alpha}\right), \alpha=\frac{1}{2}, \frac{3}{2}$, depending on the approximation along the interface lines.

In two subsequent papers, we discuss the derivation of efficient iterative methods for solving the composite grid system, extending the preconditioners developed by Bramble, Ewing, Pasciak, and Schatz [5] and by McCormick [11] and McCormick and Thomas [12] (the FAC-method) to our case and to the multilevel case.

\section{NOTATION AND PROBLEM FORMULATION}

2.1. Preliminaries and problem formulation. We use the standard notation for Sobolev spaces [1]:

$$
H^{m}=H^{m}(\Omega)=\left\{u \in L^{2}(\Omega): D^{\alpha} u \in L^{2}(\Omega),|\alpha| \leq m\right\}, \quad m \geq 0 .
$$

The norm in $H^{m}(\Omega)$ is denoted $\|\cdot\|_{m, \Omega}$ and defined by

$$
\|u\|_{m, \Omega} \equiv\left(\sum_{i=0}^{m}|u|_{i, \Omega}^{2}\right)^{2}, \quad|u|_{i, \Omega} \equiv\left(\sum_{|\alpha|=i}\left\|D^{\alpha} u\right\|_{0, \Omega}^{2}\right)^{1 / 2},
$$

where $\|\cdot\|_{0, \Omega}$ is the standard $L^{2}$-norm in $\Omega$. We also use Sobolev spaces with real index $m>0$ [1].

We consider the following mixed boundary value problem: find a function $u(x)$ which satisfies the following differential equation in a bounded domain $\Omega \subset \mathbb{R}^{2}$ :

$$
\frac{\partial W^{(1)}}{\partial x_{1}}+\frac{\partial W^{(2)}}{\partial x_{2}}=f(x), \quad x \in \Omega
$$

with

$$
W^{(1)}(x)=-a(x) \frac{\partial u}{\partial x_{1}}, \quad W^{(2)}(x)=-a(x) \frac{\partial u}{\partial x_{2}}, \quad x \in \Omega,
$$

and the boundary conditions

$$
\begin{gathered}
u(x)=g(x) \quad \text { on } \Gamma_{D}, \\
W_{\nu} \equiv-a(x) \frac{\partial u}{\partial \nu}=0 \quad \text { on } \Gamma \backslash \Gamma_{D},
\end{gathered}
$$


where $\nu$ is the unit vector normal to the boundary $\Gamma$ of $\Omega$ and $\Gamma_{D}$ is a part of $\Gamma$ with positive measure. We suppose that there are positive constants $a_{0}$ and $a_{1}$ such that

$$
0<a_{0} \leq a(x) \leq a_{1} \text { in } \Omega .
$$

We are considering a mixed boundary value problem only for the sake of simplicity of presentation. Here, we concentrate on the difficulties arising from local refinement of the mesh. We develop a general approach for overcoming two specific difficulties: constructing finite difference approximation on a composite grid and solving the corresponding system of linear equations. The latter will be studied in subsequent papers.

From the point of view of reservoir simulation, the pure Neumann boundary value problem is more realistic. Then, condition (2.5) is imposed on the whole boundary $\Gamma$. In this case, a necessary condition for existence of the solution is $\int_{\Omega} f(x) d x=0$, and a unique solution can be selected by specifying the value of $\int_{\Omega} u(x) d x$. For our approach, these details are insignificant; but since they are troublesome, we prefer to demonstrate our technique on a simpler problem and to concentrate on the difficulties arising from the local grid refinement. In order to explain our ideas and results better, we shall assume that $a(x) \equiv 1$.

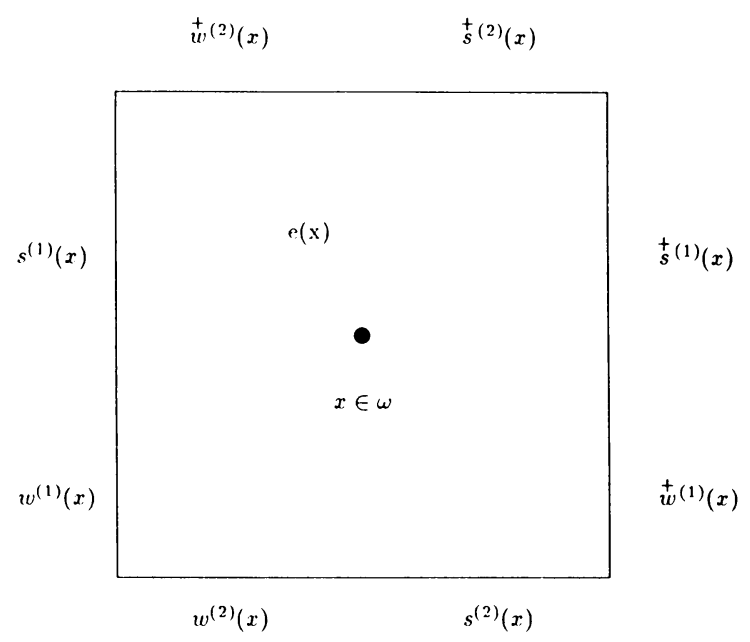

FIGURE 2.1

A grid cell

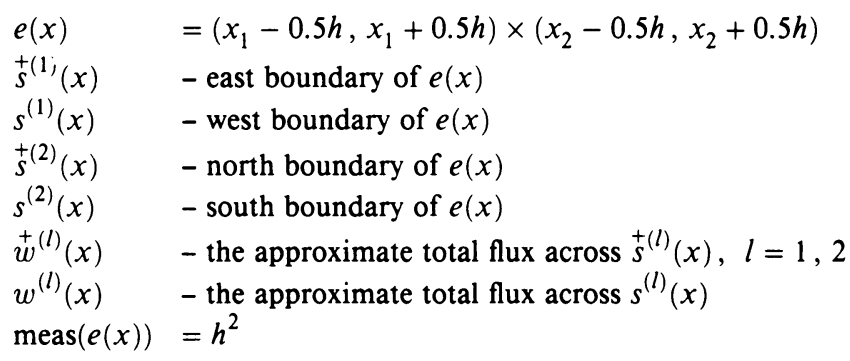


If $e=e(x)$ is the cell shown in Figure 2.1, then by integrating (2.2) over $e$ we get

$$
\int_{s^{+}(1)} W^{(1)} d s-\int_{s^{(1)}} W^{(1)} d s+\int_{s^{(2)}} W^{(2)} d s-\int_{s^{(2)}} W^{(2)} d s=\iint_{e(x)} f(t) d t
$$

which is a starting point for all of our approximations.

2.2. Grids, grid functions, and associated notation. We suppose that $\Omega$ is a rectangle with sides parallel to the axes $x_{1}$ and $x_{2}$. Extensions to the case of general domains and elliptic problems with Dirichlet boundary conditions can be accomplished using the technique described in Samarskii, Lazarov, and Makarov [18, Chapter III, p. 123].

We consider the case of cell-centered grids, which, owing to their good conservation properties, are very popular in reservoir simulation, weather prediction, heat transfer, etc. $[4,8-10,13,14,20]$. Our approximation of the differential equation is based on the finite volume approach and uses the balance equation (2.6). This approach was developed and extensively studied in the early 1960's by Tikhonov and Samarskii in a series of papers [15, 17, 19] (see also [16]) and rediscovered and augmented with new techniques in the mid 1980's (see $[9,10$, 21]).

We cover the plane $\mathbb{R}^{2}$ by square cells with sides of length $h$. The grid points are the centers of the cells. We suppose that the Dirichlet boundary $\Gamma_{D}$ passes through the grid points (as shown in Figure 2.2). Our approach is easily extended to the case of a nonuniform rectangular grid: the grid cells are rectangles of size $h_{1} \times h_{2}$, and the grid points are the centers of the cells.

Our goal is to develop a general approach for deriving finite difference approximations on a composite grid where we also introduce a refined grid along the standard grid in subregions of special interest. We denote the subregions covered by a refined grid by $\Omega_{2}$. If the remaining part of $\Omega$ is denoted by $\Omega_{1}$, then we have $\bar{\Omega}=\bar{\Omega}_{1} \cup \bar{\Omega}_{2}$.

The fine grid is introduced by subdividing the coarse grid cells in $\Omega_{2}$ into a certain number of fine grid cells and introducing as grid points the centers of the new, finer cells (see Figure 2.2). Therefore, we have cells of two different sizes: coarse grid cells of size $h_{c}$ and fine grid cells of size $h_{f}=\frac{1}{m} h_{c}, m>1$ integervalued. The letter $h$ will be used for both cases-fine and coarse cell sizes. When $m$ is an odd number, the points of the coarse grid in $\Omega_{2}$ will coincide with some of the points of the refined grid. This will be assumed throughout the paper. In this case, we say that the coarse grid is imbedded in the fine grid (see Figure 2.2).

For a given cell with center $x \in \omega$, we use the notation of Figure 2.1. We stress that $\stackrel{+}{w}^{(l)}(x)$ and $w^{(l)}(x)$ are approximations of $\int_{s^{(l)}} W^{(l)} d s$ and $\int_{s^{(l)}} W^{(l)} d s$, in (2.6), respectively.

The centers of the coarse-grid cells contained in $\Omega$ define the coarse grid, which we denote by $\tilde{\omega}$. The set of coarse-grid points in $\Omega_{2}$, we denote by $\tilde{\omega}_{2}$; 


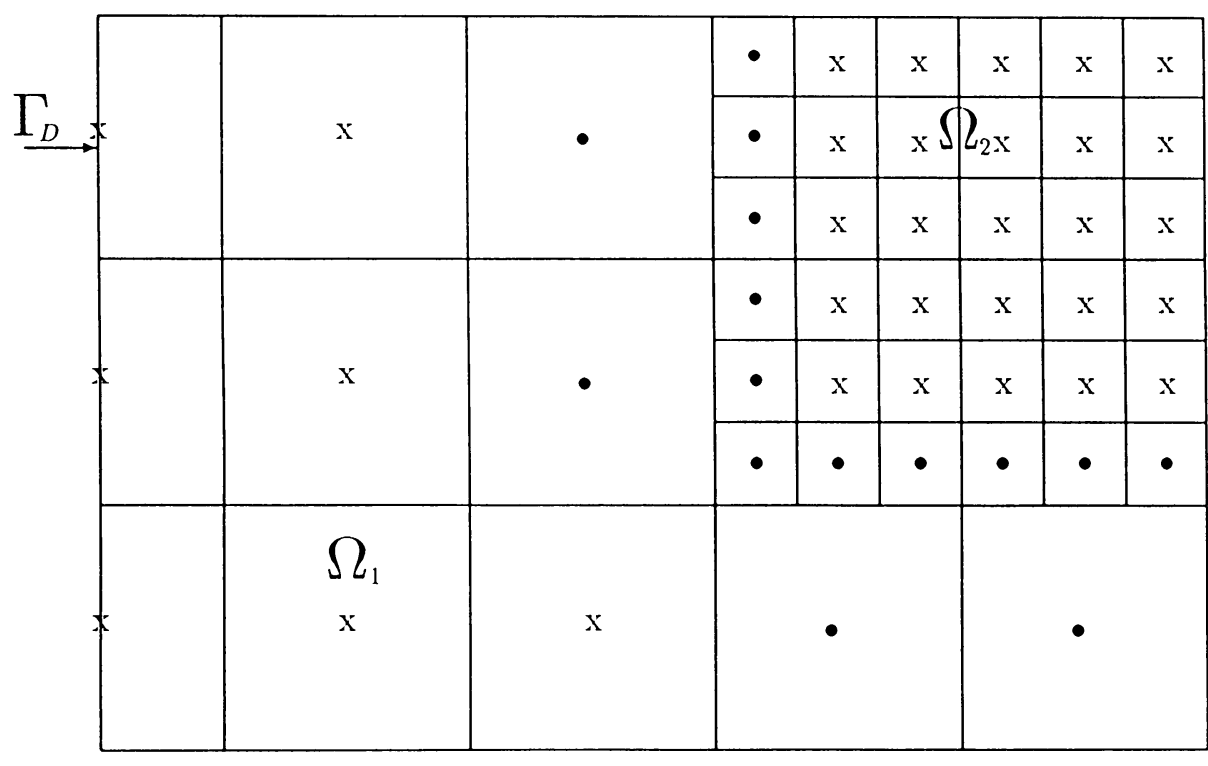

x - regular grid points,

- - irregular grid points

FIGURE 2.2

Grid with local refinement $h_{c}=3 h_{f}$

i.e., $\tilde{\omega}_{2}=\tilde{\omega} \cap \Omega_{2}$. The coarse-grid points in $\Omega_{1}$ and the fine-grid points in $\Omega_{2}$ define the composite grid, denoted by $\omega$. The set of grid points lying on $\Gamma_{D}$, we denote by $\gamma_{D}$. We call the grid points of the composite grid next to the boundary between $\Omega_{1}$ and $\Omega_{2}$ irregular. All remaining grid points are called regular. The grid points are denoted by $x=\left(x_{1}, x_{2}\right)$ or $\left(x_{1, i}, x_{2, j}\right)$, where $i, j$ are integer indices.

Functions $y(x)$ of a discrete argument $x \in \omega$ are called grid functions. We consistently use the dual notation for the value of the function $y$ at the grid point $x=\left(x_{1, i}, x_{2, j}\right) ; y(x)=y\left(x_{1, i}, x_{2, j}\right)=y_{i, j}$. For a given grid function $y(x), x \in \omega$, we use the following discrete $L^{2}$-norm:

$$
\|y\|_{0, \omega}=\left(\sum_{x \in \omega} y^{2}(x)\right)^{1 / 2} .
$$

On the other hand, the grid function $y(x)$ can be considered as an element of a vector space of dimension equal to $N$, the number of the grid points in $\omega$. In this case, we denote $y(x)$ as $\mathbf{y} \in \mathbb{R}^{N}$ and consider it as an $N$-dimensional column vector. Then $\mathbf{y}^{T}$ will be the row vector transpose of $\mathbf{y}$.

\section{Finite DifferenCE APPROXIMATIONS}

In this section, we derive three finite difference approximations of the problem (2.2)-(2.5) on a composite cell-centered grid. First, we consider the regular 
grid points, and following [16], derive a 5-point-star approximation with coefficients some harmonic mean values of $a(x)$. Next, we treat the irregular grid points and propose three different approximations of the fluxes-simple symmetric, nonsymmetric, and more accurate symmetric approximations. Then, we formulate the corresponding discrete problems and investigate the algebraic properties of their matrices. We prove that all three approximations lead to systems with invertible matrices and, moreover, that the matrices are spectrally equivalent to the matrix of the simplest symmetric approximation. For the nonsymmetric case, this is explained below.

3.1. Approximation of the balance equation. A starting point for the finite difference approximation of (2.2)-(2.5) is the balance equation (2.6). According to our notation (see Figure 2.1), $w^{(l)}(x)$ and $\stackrel{+}{w}^{(l)}(x)$ are approximate fluxes across the faces $s^{(l)}(x)$ and $s^{+(l)}(x), l=1,2$, of a given cell $e(x), x \in \omega$. Then, replacing $\int W^{(l)} d s$ by their approximations, we get the following difference equations (in terms of approximate fluxes):

$$
\stackrel{+}{w}^{(1)}(x)-w^{(1)}(x)+\stackrel{+}{w}^{(2)}(x)-w^{(2)}(x)=\iint_{e(x)} f(t) d t \equiv \varphi(x),
$$

a discrete analog of the balance equation (2.6). In order to complete the finite difference approximation, we have to find a finite difference approximation of the relations $(2.3)$; i.e., we have to express the approximate fluxes $w^{(l)}(x)$, $\stackrel{+}{w}^{(l)}(x), l=1,2$, by the approximate values $y(x)$ of the pressure $u(x)$ at the grid points.

Let us note first that if any of the boundaries of the cell $e(x)$ lie on $\Gamma \backslash \Gamma_{D}$, then on this boundary we have $W_{\nu}(x)=0$; therefore, it is natural to assume that the corresponding approximate flux is zero. This is equivalent to an even extension with respect to the boundary of our grid functions outside $\Omega$. With this convention, we proceed with the approximation of the fluxes at the regular grid points (see Figure 3.1).

Since $a(x)$ is strictly positive, we can rewrite (2.3) in the form

$$
\frac{\partial u}{\partial x_{l}}=-\frac{W^{(l)}(x)}{a(x)}, \quad x \in \Omega, l=1,2 .
$$

Now, let us integrate this equation for $l=1$ along the interval with endpoints $\left(x_{1, i-1}, x_{2, j}\right)$ and $\left(x_{1, i}, x_{2, j}\right)$. We get

(3.3) $u_{i, j}-u_{i-1, j}=-\int_{x_{1, i-1}}^{x_{1, i}} \frac{W^{(1)}\left(s, x_{2, j}\right)}{a\left(s, x_{2, j}\right)} d s \cong-W_{i-1 / 2, j}^{(1)} \int_{x_{1, i-1}}^{x_{1, i}} \frac{d s}{a\left(s, x_{2, j}\right)}$, where by $W_{i-1 / 2, j}^{(1)}$ we mean $W^{(1)}\left(x_{1, i-1}+\frac{1}{2} h, x_{2, j}\right)$. 


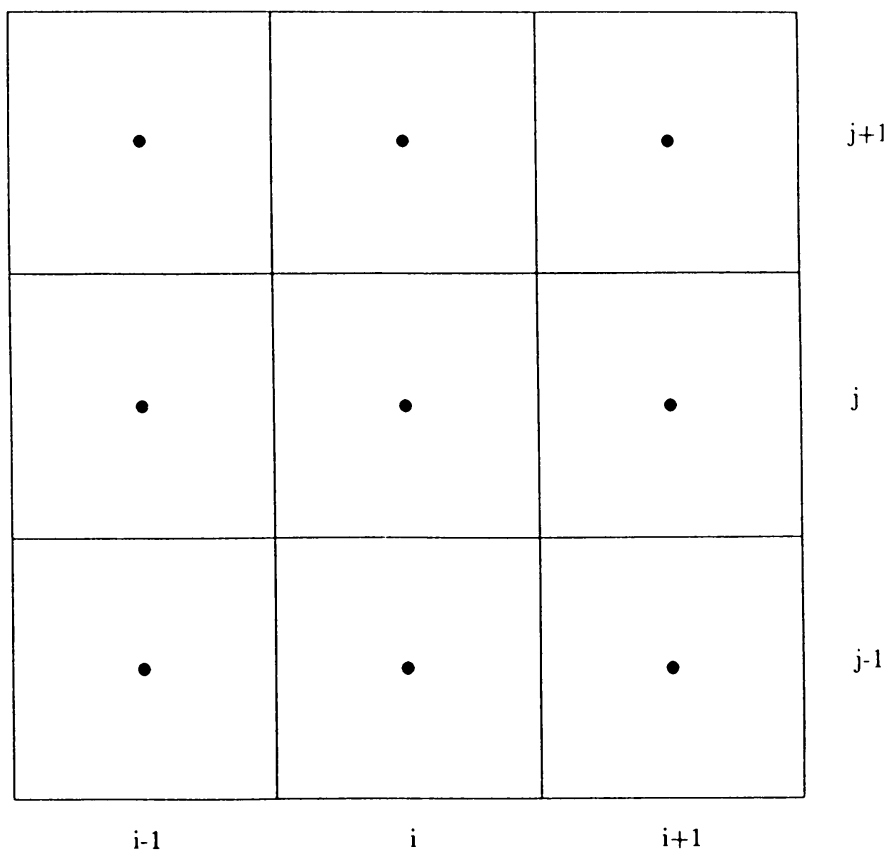

FIGURE 3.1

Regular grid point $\left(x_{1, i}, x_{2, j}\right)$

We can now write the following approximate relations, which will be a basis for the finite difference approximation:

$$
\begin{aligned}
\int_{s^{(1)}} W^{(1)}(x) d s & \cong h W_{i-1 / 2, j}^{(1)} \\
& \cong-\left(\frac{1}{h} \int_{x_{1, i-1}}^{x_{1, i}} \frac{d s}{a\left(s, x_{2, j}\right)}\right)^{-1}\left[u_{i, j}-u_{i-1, j}\right], \\
\int_{s^{(2)}} W^{(2)}(x) d s & \cong h W_{i, j-1 / 2}^{(2)} \\
& \cong-\left(\frac{1}{h} \int_{x_{2, j-1}}^{x_{2, j}} \frac{d s}{a\left(x_{1, i}, s\right)}\right)^{-1}\left[u_{i, j}-u_{i, j-1}\right] .
\end{aligned}
$$

These approximate relations show how to link the approximate fluxes $w^{(l)}(x)$, $l=1,2$, with the approximate values $y(x)$ of the pressure. Specifically, at any regular point $x \in \omega$, we define

$$
\begin{array}{ll}
w^{(l)}(x) \equiv w_{i, j}^{(l)}=-k_{i, j}^{(l)} \bar{\Delta}_{l} y_{i, j} \equiv-k^{(l)} \bar{\Delta}_{l} y, & l=1,2, \\
\stackrel{+}{w}^{(l)}(x) \equiv \stackrel{+}{w}_{i, j}^{(l)}=-\stackrel{+}{k}_{i, j}^{(l)} \Delta_{l} y_{i, j} \equiv-\dot{+}^{(l)} \Delta_{l} y, & l=1,2,
\end{array}
$$


where

$$
\begin{aligned}
& k_{i, j}^{(1)}=\left(\frac{1}{h} \int_{x_{1, i-1}}^{x_{1, i}} \frac{d s}{a\left(s, x_{2, j}\right)}\right)^{-1}, \quad \stackrel{+}{k}_{i, j}^{(1)}=k_{i+1, j}^{(1)}, \\
& k_{i, j}^{(2)}=\left(\frac{1}{h} \int_{x_{2, j-1}}^{x_{2, j}} \frac{d s}{a\left(x_{1, i}, s\right)}\right)^{-1}, \quad \stackrel{+}{k}_{i, j}^{(2)}=k_{i, j+1}^{(2)}, \\
& \bar{\Delta}_{1} y_{i, j}=y_{i, j}-y_{i-1, j}, \quad \Delta_{1} y_{i, j}=y_{i+1, j}-y_{i, j}, \\
& \bar{\Delta}_{2} y_{i, j}=y_{i, j}-y_{i, j-1}, \quad \Delta_{2} y_{i, j}=y_{i, j+1}-y_{i, j} .
\end{aligned}
$$

Then inserting (3.5) into (3.1), we get the desired finite difference approximation of equation (2.2) at the regular grid points.

Remark 3.1. One can see easily how the derived approximation could be extended to the case of rectangular cells on a nonuniform mesh. If, instead of the uniform grid parameter $h$, we introduce $\left(h_{1, i}, h_{2, j}\right)$-the sizes of the cell $e_{i, j}$ in the $x_{1}$ and $x_{2}$ directions - then formulas (3.5) and (3.6) will have the following form:

$$
\begin{gathered}
w^{(1)}=-\frac{2 h_{2, j}}{h_{1, i}+h_{1, i-1}} k^{(1)} \bar{\Delta}_{1} y, \\
k^{(1)}=\left(\frac{2}{h_{1, i}+h_{1, i-1}} \int_{x_{1, i-1}}^{x_{1, i}} \frac{d s}{a\left(s, x_{2, j}\right)}\right)^{-1},
\end{gathered}
$$

and similarly for $\stackrel{+}{w}^{(1)}, w^{(2)}$, and $\stackrel{+}{w}^{(2)}$.

Remark 3.2. Obviously, at the regular grid points, we have

$$
\stackrel{+}{w}_{i, j}^{(1)}=w_{i+1, j}^{(1)} \quad \text { and } \quad \stackrel{+}{w}_{i, j}^{(2)}=w_{i, j+1}^{(2)} \text {. }
$$

Then equation (3.1) can be written in the form

$$
\sum_{l=1}^{2} \Delta_{l} w^{(l)}=\iint_{e(x)} f(t) d t, \quad x \in \omega \text { a regular point. }
$$

Remark 3.3. The coefficients $k^{(l)}$ and $\stackrel{+}{k}^{(l)}, l=1,2$, are called harmonic mean approximations of $a(x)$, and are frequently used in the reservoir simulation literature (see [4, p. 84] and also [8, 13]). This approximation is particularly useful when $a(x)$ is a piecewise smooth function and can be derived from the lowestorder mixed finite element method with "lumping the mass" (see, for example, [21]). If $a(x)$ is a smooth function in $\Omega$, then appropriate approximations to $k_{i, j}^{(1)}$ and $\stackrel{+}{k}_{i, j}^{(1)}$ are $a\left(x_{1, i-1 / 2}, x_{2, j}\right)$ and $a\left(x_{1, i+1 / 2}, x_{2, j}\right)$, respectively, with $x_{1, i \pm 1 / 2}=x_{1, i} \pm \frac{1}{2} h$.

Remark 3.4. If $a(x) \equiv 1$, then (3.5) is the standard 5-point approximation of the Poisson equation with right-hand side the average of $f(x)$ over the cell $e(x)$. If $f(x)$ is a smooth function, then the integral can be approximated 


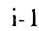

$\mathrm{i}+1$

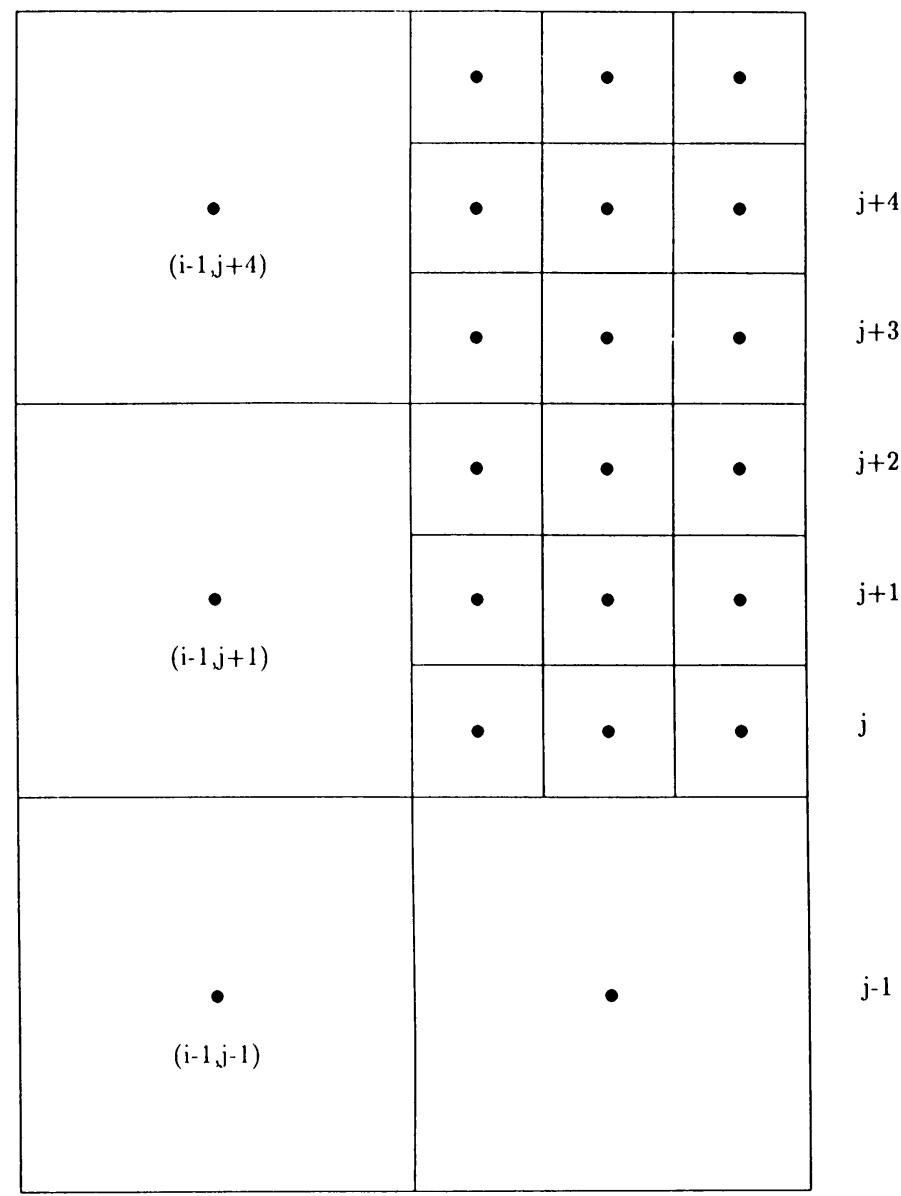

FIGURE 3.2

Irregular grid point $\left(x_{1, i-1}, x_{2, j+1}\right)$

by the midpoint quadrature rule. But, we prefer to keep the right-hand side as an integral of $f(x)$, so that we keep an open option for nonsmooth $f(x)$. However, if we use any approximation of the integral, then we have to add its error to the local truncation error of the difference scheme (see the presentation of (4.2) and the estimate (4.10) below).

We next consider the approximation of the fluxes at the irregular points (see Figure 3.2). Our ultimate requirement is that the finite difference scheme conserve mass. Since (in the particular situation shown on Figure 3.2)

$$
\int_{s_{i-1, j+1}^{(1)}} W^{(1)} d s=\int_{s_{i, j}^{(1)}} W^{(1)} d s+\int_{s_{i, j+1}^{(1)}} W^{(1)} d s+\int_{s_{i, j+2}^{(1)}} W^{(1)} d s,
$$


mass conservation requires that the approximate fluxes satisfy

$$
\stackrel{+}{w}_{i-1, j+1}^{(1)}=w_{i, j}^{(1)}+w_{i, j+1}^{(1)}+w_{i, j+2}^{(1)} \text {. }
$$

Thus, our task now is to find an appropriate approximation of the fluxes $w_{i, j+l}^{(1)}$, $l=0,1,2$. A natural approximation of the fluxes across the boundaries $s_{i, j+l}^{(1)}$ uses the values of the pressure at the points $\left(x_{1, i-1}, x_{2, j+l}\right)$, which for $l=0,2$ are not grid points. The needed values could be obtained from the values at the coarse grid points by piecewise polynomial interpolation. Below, we use piecewise constant and piecewise linear interpolation.

3.2. Simple symmetric approximation. We consider the case of irregular cell $e_{i, j}$ shown in Figure 3.2. Applying (3.3) and (3.4) in this case, we get

$$
\int_{s_{i, j}^{(1)}} W^{(1)} d s \cong-h_{f}\left(\int_{x_{1, i-1}}^{x_{1, i}} \frac{d s}{a\left(s, x_{2, j}\right)}\right)^{-1}\left(u_{i, j}-u_{i-1, j}\right) .
$$

Since we would like to have a coefficient $k^{(1)}$ which does not depend on $h$, we divide the corresponding integral by the length of the interval of integration $\left(x_{1, i-1}, x_{1, i}\right)$ and denote it by

$$
k_{i, j}^{(1)}=\left(\frac{2}{h_{c}+h_{f}} \int_{x_{1, i-1}}^{x_{1, i}} \frac{d s}{a\left(s, x_{2, j}\right)}\right)^{-1} .
$$

Since the point $\left(x_{1, i-1}, x_{2, j}\right)$ is not a grid point, we can assume that the approximate solution is extended over the cell $e_{i-1, j}$ as a constant and therefore we can put $y_{i-1, j} \cong y_{i-1, j+1}$. Thus, we get the following simplest form for the approximate fluxes $w_{i, j+l}^{(1)}, l=0,1,2$ :

$$
w_{i, j+l}^{(1)}=-\frac{2 h_{f}}{h_{c}+h_{f}} k_{i, j+l}^{(1)}\left(y_{i, j+l}-y_{i-1, j+1}\right) \equiv-\frac{2 h_{f}}{h_{c}+h_{f}} k_{i, j+l}^{(1)} \bar{\Delta}_{1} v_{i, j+l},
$$

where, implicitly, we have defined

$$
\begin{aligned}
\bar{\Delta}_{1} y_{i, j+l} & =y_{i, j+l}-y_{i-1, j+1}, \\
k_{i, j+l}^{(1)} & =\left(\frac{2}{h_{c}+h_{f}} \int_{x_{1, i-1}}^{x_{1, i}} \frac{d s}{a\left(s, x_{2, j+l}\right)}\right)^{-1}
\end{aligned}
$$

at the irregular points $\left(x_{1, i}, x_{2, j+l}\right), l=0,1,2$.

Using (3.10), we define the flux $\stackrel{+}{w}_{i-1, j+1}^{(1)}$ by (3.8). Similarly, we define the approximate fluxes at the irregular grid points $\left(x_{1, i+l}, x_{2, j}\right), l=0,1,2$ (see Figure 3.2):

$$
\begin{aligned}
& w_{i+l, j}^{(2)}=-\frac{1}{2} k_{i+l, j}^{(2)}\left(y_{i+l, j}-y_{i+1, j-1}\right) \equiv-\frac{1}{2} k_{i+l, j}^{(2)} \bar{\Delta}_{2} y_{i+l, j}, \\
& k_{i+l, j}^{(2)}=\left(\frac{2}{h_{c}+h_{f}} \int_{x_{2, j-1}}^{x_{2, j}} \frac{d s}{a\left(x_{1, i+l}, s\right)}\right)^{-1}, \quad l=0,1,2 .
\end{aligned}
$$


Note that in (3.11) we use the fact that $2 h_{f} /\left(h_{f}+h_{c}\right)=\frac{1}{2}$ for the particular situation of Figure 3.2.

For the approximation of the remaining fluxes at the irregular points, we can use the approximations in (3.5) and (3.6).

Remark 3.5. Here we have considered the case $h_{c}=3 h_{f}$ only for definiteness. It is easy to extend this approximation to the general case $h_{c}=m h_{f}$. Then, (3.9) will have the form

$$
w_{i, j+l}^{(1)}=-\frac{2}{m+1} k_{i, j+l}^{(1)} \bar{\Delta}_{1} y_{i, j+l}, \quad l=0,1, \ldots, m-1,
$$

with $k^{(1)}$ and $\bar{\Delta}_{1}$ defined by (3.10). Similarly, we can define $w^{(2)}$ and $k^{(2)}$.

Remark 3.6. It is easy to see that this approximation leads to a system of linear equations with a symmetric matrix. Since we have used piecewise constant interpolation, it is natural to expect that the approximation properties of the finite difference scheme are quite poor. We show that the rate of convergence in the discrete energy norm, defined below by (4.9), of this scheme is $O\left(h^{1 / 2}\right)$. Therefore, it is natural to look for a better approximation; this is the main concern of the following two subsections.

3.3. Nonsymmetric approximation. In this case, we suppose that $y(x)$ is interpolated linearly between any two neighboring coarse-grid nodes. For example, the values $y_{i-1, j+l}, l=0,2$ (see Figure 3.2 ), are

$$
y_{i-1, j}=\frac{2}{3} y_{i-1, j+1}+\frac{1}{3} y_{i-1, j-1}
$$$$
y_{i-1, j+2}=\frac{2}{3} y_{i-1, j+1}+\frac{1}{3} y_{i-1, j+4} \text {. }
$$

Then, instead of (3.9), we have the following expressions for the approximate fluxes:

$$
\begin{aligned}
w_{i, j}^{(1)} & =-\frac{1}{2} k_{i, j}^{(1)}\left(y_{i, j}-\frac{2}{3} y_{i-1, j+1}-\frac{1}{3} y_{i-1, j-1}\right), \\
w_{i, j+2}^{(1)} & =-\frac{1}{2} k_{i, j+2}^{(1)}\left(y_{i, j+2}-\frac{2}{3} y_{i-1, j+1}-\frac{1}{3} y_{i-1, j+4}\right) .
\end{aligned}
$$

Taking into account formulas (3.6) and (3.10), we can rewrite these expressions in the following form:

$$
\begin{aligned}
w_{i, j}^{(1)} & =-\frac{1}{2} k_{i, j}^{(1)} \bar{\Delta}_{1} y_{i, j}-\frac{1}{6} k_{i, j}^{(1)} \bar{\Delta}_{2} y_{i-1, j+1}, \\
w_{i, j+1}^{(1)} & =-\frac{1}{2} k_{i, j+1}^{(1)} \bar{\Delta}_{1} y_{i, j+1}, \\
w_{i, j+2}^{(1)} & =-\frac{1}{2} k_{i, j+2}^{(1)} \bar{\Delta}_{1} y_{i, j+2}+\frac{1}{6} k_{i, j+2}^{(1)} \Delta_{2} y_{i-1, j+1} .
\end{aligned}
$$

Comparing (3.9) with (3.14), we see that in the latter case a correction proportional to $\frac{1}{2} h a \frac{\partial u}{\partial x_{2}}$ has been added to the approximate fluxes. If the point $\left(x_{1, i-1}, x_{2, j+1}\right)$ is next to the north boundary of $\Omega$, then $\left(x_{1, i-1}, x_{2, j+4}\right)$ is outside $\Omega$, and the corresponding correction is not available. But since $u(x)$ satisfies (2.5), $\frac{\partial u}{\partial x_{2}}=O(h)$ near the boundary, and we can skip the correction. Thus, near the boundary we can adopt the approximation of the simplest symmetric scheme. In the case of boundary condition $a \frac{\partial u}{\partial x_{2}}=\mu$, we have to add $\frac{1}{2} h_{f} \mu(x)$ to the right-hand side of the difference scheme. 


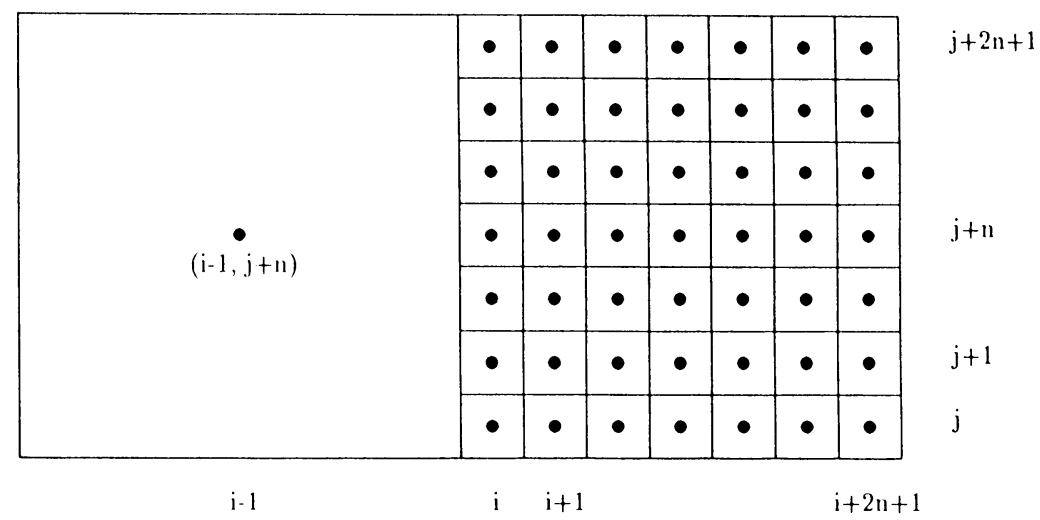

FIGURE 3.3

Irregular grid cell with $h_{c}=(2 n+1) h_{f}$

Similarly, we have the following expressions for the approximate fluxes at the irregular points $\left(x_{1, i+l}, x_{2, j}\right), l=0,1,2$ :

$$
\begin{aligned}
w_{i, j}^{(2)} & =-\frac{1}{2} k_{i, j}^{(2)} \bar{\Delta}_{2} y_{i, j}-\frac{1}{6} k_{i, j}^{(2)} \bar{\Delta}_{1} y_{i+1, j-1}, \\
w_{i+1, j}^{(2)} & =-\frac{1}{2} k_{i+1, j}^{(2)} \bar{\Delta}_{2} y_{i+1, j}, \\
w_{i+2, j}^{(2)} & =-\frac{1}{2} k_{i+2, j}^{(2)} \bar{\Delta}_{2} y_{i+2, j}+\frac{1}{6} k_{i+2, j}^{(2)} \Delta_{1} y_{i+1, j-1} .
\end{aligned}
$$

For definiteness, we have considered the case when $h_{c}=3 h_{f}$. In the case of refinement $h_{c}=(2 n+1) h_{f}$ (shown in Figure 3.3), the corresponding formulas for the approximate fluxes are derived in a similar manner. For example, instead of (3.14), we have

$$
\begin{array}{r}
w_{i, j+n+l}^{(1)}=-\frac{k_{i, j+n+l}^{(1)}}{n+1}\left[\bar{\Delta}_{1} y_{i, j+n+l}-\frac{l}{2 n+1} \Delta_{2} y_{i-1, j+n}\right], \\
l=1,2, \ldots, n, \\
w_{i, j+n+l}^{(1)}=-\frac{k_{i, j+n+l}^{(1)}}{n+1}\left[\bar{\Delta}_{1} y_{i, j+n+l}-\frac{l}{2 n+1} \bar{\Delta}_{2} y_{i-1, j+n}\right], \\
l=-1,-2, \ldots,-n .
\end{array}
$$

3.4. More accurate symmetric approximation. The approximate fluxes in (3.14) are sums of two terms: the first one is exactly the symmetric flux (3.9), the second a correction, approximately equal to $\pm \frac{h_{f}}{2} a \frac{\partial u}{\partial x_{2}}$, that improves the approximation. Unfortunately, this correction leads to a nonsymmetric scheme. In order to produce a symmetric scheme which has the same approximation properties as the nonsymmetric one, we have to replace the second terms in (3.14) by terms which approximate $\frac{\partial u}{\partial x_{2}}$ and give a symmetric scheme. This can be done in several ways. If we replace them by

$$
\pm \frac{1}{4} k_{i, j+1}^{(1)}\left(y_{i, j+2}-y_{i, j}\right) \text {, }
$$


then we get the following approximation of the fluxes:

$$
\begin{aligned}
w_{i, j}^{(1)} & =-\frac{1}{2} k_{i, j}^{(1)} \bar{\Delta}_{1} y_{i, j}-\frac{1}{4} k_{i, j+1}^{(1)}\left(y_{i, j+2}-y_{i, j}\right), \\
w_{i, j+2}^{(1)} & =-\frac{1}{2} k_{i, j+2}^{(1)} \bar{\Delta}_{1} y_{i, j+2}+\frac{1}{4} k_{i, j+1}^{(1)}\left(y_{i, j+2}-y_{i, j}\right) .
\end{aligned}
$$

Similarly, we get the following approximation of the fluxes $w^{(2)}$ at the irregular points $\left(x_{1, i}, x_{2, j}\right)$ and $\left(x_{1, i+2}, x_{2, j}\right)$ :

$$
\begin{aligned}
w_{i, j}^{(2)} & =-\frac{1}{2} k_{i, j}^{(2)} \bar{\Delta}_{2} y_{i, j}-\frac{1}{4} k_{i+1, j}^{(2)}\left(y_{i+2, j}-y_{i, j}\right), \\
w_{i+2, j}^{(2)} & =-\frac{1}{2} k_{i, j}^{(2)} \bar{\Delta}_{2} y_{i+2, j}+\frac{1}{4} k_{i+1, j}^{(2)}\left(y_{i+2, j}-y_{i, j}\right) .
\end{aligned}
$$

In the general case $h_{c}=(2 n+1) h_{f}$ (shown in Figure 3.3), we get the following approximations of the fluxes for the more accurate symmetric scheme:

$$
\begin{array}{r}
w_{i, j+n+l}^{(1)}=-\frac{1}{n+1}\left[k_{i, j+n+l}^{(1)} \bar{\Delta}_{1} y_{i, j+n+l}-\frac{k_{i, j+n}^{(1)}}{2}\left(y_{i, j+n+l}-y_{i, j+n-l}\right)\right], \\
l= \pm 1, \pm 2, \ldots, \pm n .
\end{array}
$$

The case $h_{c}=2 n h_{f}$ is treated in the same way.

3.5. Formulation of the discrete problem. Summarizing these approximations, we formulate the following three finite difference schemes for the problem (2.2)-(2.5): find a grid function $y(x)$ which satisfies the finite difference equations (on the composite grid)

$$
-\sum_{l=1}^{2}\left(\stackrel{+}{w}^{(l)}(x)-w^{(l)}(x)\right)=\iint_{e(x)} f(t) d t \equiv \varphi(x) \text { on } \omega
$$

and the Dirichlet boundary condition

$$
y(x)=g(x) \text { on } \Gamma_{D},
$$

where the approximate fluxes $w^{(l)}(x)$ and $\stackrel{+}{w}^{(l)}(x)$ are defined by (3.5) and (3.6) at all regular grid points and by any of the formulas (3.9)-(3.11), or (3.14), or (3.15) at the irregular points.

As we mentioned above, the grid function $y(x), x \in \omega$, can be considered as a column vector $\mathbf{y} \in \mathbb{R}^{N}$, where $N$ is the number of the grid points in $\omega$. Then any of the three finite difference schemes can be written as a system of linear algebraic equations

$$
A \mathbf{y}=\mathbf{f}
$$

where in the right-hand side $\mathbf{f}$ we have taken the boundary condition (3.17) into account.

Let

$$
A_{0} \mathbf{y}=\mathbf{f}
$$




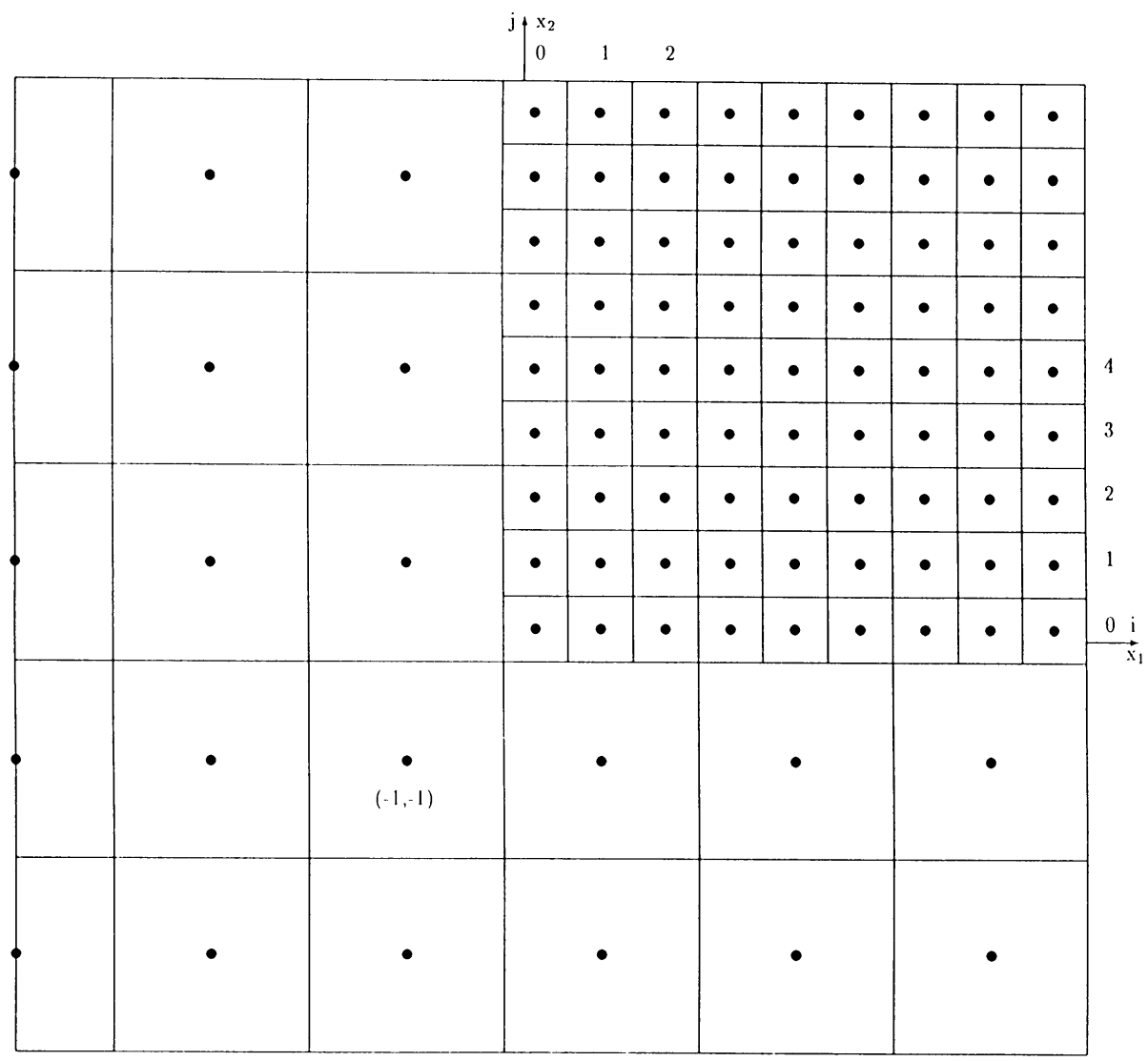

FIGURE 3.4

Composite grid in a rectangle

be the matrix notation for the finite difference scheme (3.16), (3.17), (3.5), (3.6), and (3.9)-(3.11); that is, $A_{0}$ is a matrix of the simplest symmetric scheme.

Now we shall prove that the matrix $A$ of $(3.18)$ is invertible, and it will follow that the finite difference scheme (3.16), (3.17) has a unique solution for any $\varphi(x), x \in \omega$, and $g(x), x \in \Gamma_{D}$. For definiteness, we shall consider the case of the grid shown on Figure 3.4.

Let us form the inner product $\mathbf{v}^{T} A \mathbf{y}$, where $A$ is defined by (3.16) and $v(x)$ is an arbitrary grid function satisfying $v(x)=0$ on $\Gamma_{D}$. Then

$$
\begin{aligned}
\mathbf{v}^{T} A \mathbf{y} & =-\sum_{x \in \omega} v(x) \sum_{l=1}^{2}\left(\stackrel{+}{w}^{(l)}(x)-w^{(l)}(x)\right) \\
& =-\sum_{l=1}^{2} \sum_{x \in \omega} v(x)\left(\stackrel{+}{w}^{(l)}(x)-w^{(l)}(x)\right) \equiv-\sum_{l=1}^{2} I_{l} .
\end{aligned}
$$

We transform the sums in (3.20), for $l=1,2$, using the following simple formula for summation by parts: let $\mathbf{v}=\left(v_{0}, v_{1}, \ldots, v_{n}\right)^{T}$ and 
$\mathbf{w}=\left(w_{0}, w_{1}, \ldots, w_{n}\right)^{T}$ be vectors in $\mathbb{R}^{n+1} ;$ then

$$
\sum_{i=1}^{n-1}\left(w_{i+1}-w_{i}\right) v_{i}=-\sum_{i=1}^{n-1} w_{i}\left(v_{i}-v_{i-1}\right)+w_{n} v_{n-1}-w_{1} v_{0}
$$

In order to use this formula for the case of Figure 3.4, we present the term $I_{1}$ in the form

$$
I_{1}=\sum_{j<0} \sum_{\text {all } i}+\sum_{j>0} \sum_{i<0}+\sum_{j \geq 0} \sum_{i \geq 0} .
$$

Then, using formula (3.21) for each sum and taking into account that $v(x)=$ 0 for $x \in \Gamma_{D}$, we get

$$
\sum_{j<0} \sum_{\text {all } i}\left(\stackrel{+}{w}_{i, j}^{(1)}-w_{i, j}^{(1)}\right) v_{i, j}=-\sum_{j<0} \sum_{i} w_{i, j}^{(1)}\left(v_{i, j}-v_{i-1, j}\right)
$$

where we have taken into account that for $j<0$, the approximate flux $w_{i, j}^{(1)}$ is defined as at the regular points and $\stackrel{+}{w}_{j, n-1}^{(1)}=0$. Similarly,

$$
\begin{aligned}
& \sum_{j>0} \sum_{i<0}\left(\stackrel{+}{w}_{i, j}^{(1)}-w_{i, j}^{(1)}\right) v_{i, j} \\
& \quad=-\sum_{j>0}\left\{\sum_{i<0} w_{i, j}^{(1)}\left(v_{i, j}-v_{i-1, j}\right)-\stackrel{+}{w}_{-1, j}^{(1)} v_{-1, j}\right\}
\end{aligned}
$$

and

$$
\sum_{j \geq 0} \sum_{i \geq 0}\left(\stackrel{+}{w}_{i, j}^{(1)}-w_{i, j}^{(1)}\right) v_{i, j}=-\sum_{j \geq 0}\left\{\sum_{i \geq 1} w_{i, j}^{(1)}\left(v_{i, j}-v_{i-1, j}\right)+w_{0, j}^{(1)} v_{0, j}\right\}
$$

Note that in (3.23), we have $j=1,4,7, \ldots$, whereas in $(3.24), j=0,1$, $2, \ldots$.

Since, by (3.8),

$$
\stackrel{+}{w}_{-1, j}^{(1)}=w_{0, j}^{(1)}+w_{0, j+1}^{(1)}+w_{0, j-1}^{(1)},
$$

and taking into account the definition of $\bar{\Delta}_{1}$ from (3.6) and (3.10), we get

$$
\begin{aligned}
I_{1}= & -\sum_{\left(x_{1, i} x_{2, j}\right) \in \omega, i \neq 0} w_{i, j}^{(1)} \bar{\Delta}_{1} v_{i, j} \\
& -\sum_{j>0}\left\{w_{0, j-1}^{(1)} \bar{\Delta}_{1} v_{0, j-1}+w_{0, j}^{(1)} \bar{\Delta}_{1} v_{0, j}+w_{0, j+1}^{(1)} \bar{\Delta}_{1} v_{0, j+1}\right\} .
\end{aligned}
$$

In a similar way, we also transform the term $I_{2}$ :

$$
\begin{aligned}
I_{2}= & -\sum_{\left(x_{1, i}, x_{2, j}\right) \in \omega, j \neq 0} w_{i, j}^{(2)} \bar{\Delta}_{2} v_{i, j} \\
& -\sum_{i>0}\left\{w_{i-1,0}^{(2)} \bar{\Delta}_{2} v_{i-1,0}+w_{i, 0}^{(2)} \bar{\Delta}_{2} v_{i, 0}+w_{i+1,0}^{(2)} \bar{\Delta}_{2} v_{i+1,0}\right\} .
\end{aligned}
$$


Inserting these two identities into (3.20), we get the following basic representation:

$$
\mathbf{v}^{T} A \mathbf{y}=-\sum_{\omega}\left\{w^{(1)} \bar{\Delta}_{1} v+w^{(2)} \bar{\Delta}_{2} v\right\}
$$

which is valid for the three finite difference schemes.

Now we consider consecutively the three difference schemes based on the three approximations of the fluxes. In order to simplify our considerations, we suppose that $a(x) \equiv 1$.

Let us consider first the simplest symmetric scheme, where the approximate fluxes are defined by (3.5), (3.6), (3.9)-(3.11). By using the corresponding expressions for $w_{i, j}^{(l)}, l=1,2$, for $\left(x_{1, i}, x_{2, j}\right) \in \omega$, in (3.27), we have

$$
\mathbf{v}^{T} A_{0} \mathbf{y}=\sum_{\omega}\left(\alpha^{(1)} \bar{\Delta}_{1} y \bar{\Delta}_{1} v+\alpha^{(2)} \bar{\Delta}_{2} y \bar{\Delta}_{2} v\right)
$$

where

$$
\begin{aligned}
& \alpha^{(1)}=\alpha_{i, j}^{(1)}= \begin{cases}\frac{1}{2} & \text { for } j \geq 0, i=0, \\
1 & \text { for the remaining indices },\end{cases} \\
& \alpha^{(2)}=\alpha_{i, j}^{(2)}= \begin{cases}\frac{1}{2} & \text { for } i \geq 0, j=0, \\
1 & \text { for the remaining indices. }\end{cases}
\end{aligned}
$$

From this representation, we see that the expression $\mathbf{v}^{T} A_{0} \mathbf{y}$ is a symmetric bilinear form of $\mathbf{v}$ and $\mathbf{y}$, and that, therefore, the matrix $A_{0}$ is symmetric. If $\mathbf{v}=\mathbf{y}$, then $\mathbf{y}^{T} A_{0} \mathbf{y}$ is a sum of squared finite differences, and $\mathbf{y}^{T} A_{0} \mathbf{y}=0$ if and only if $\mathbf{y}$ is a constant vector. But since $y(x)=0$ on $\Gamma_{D}$, then $\mathbf{y}$ can only be the zero vector. Therefore the matrix $A_{0}$ is positive definite.

Remark 3.7. There exist positive constants $c_{0}$ and $c_{1}$, independent of $h$ and $y$, such that

$$
c_{0} \sum_{x \in \omega} y^{2}(x) \text { meas } e(x) \leq \mathbf{y}^{T} A_{0} \mathbf{y} \leq c_{1} \mathbf{y}^{T} \mathbf{y}=c_{1} \sum_{x \in \omega} y^{2}(x) .
$$

This shows that the condition number of $A_{0}$ is $O\left(h^{-2}\right)$.

Now let us consider the nonsymmetric approximation of the fluxes at the irregular grid points given by (3.14). Inserting (3.14) and (3.14') into (3.27), we get

$$
\begin{aligned}
\mathbf{v}^{T} A \mathbf{y}= & \sum_{\omega}\left(\alpha^{(1)} \bar{\Delta}_{1} y \bar{\Delta}_{1} v+\alpha^{(2)} \bar{\Delta}_{2} y \bar{\Delta}_{2} v\right) \\
& +\frac{1}{6} \sum_{j=1,4,7, \ldots}\left\{\bar{\Delta}_{2} y_{-1, j} \bar{\Delta}_{1} v_{0, j-1}-\Delta_{2} y_{-1, j} \bar{\Delta}_{1} v_{0, j+1}\right\} \\
& +\frac{1}{6} \sum_{i=1,4,7, \ldots}\left\{\bar{\Delta}_{1} y_{i,-1} \bar{\Delta}_{2} v_{i-1,0}-\Delta_{1} y_{i,-1} \bar{\Delta}_{2} v_{i+1,0}\right\}
\end{aligned}
$$


where $\alpha^{(l)}=\alpha_{i, j}^{(l)}, l=1,2$, are defined by (3.29). It is obvious that the bilinear form $\mathbf{v}^{T} A \mathbf{y}$ is not symmetric. Applying the Cauchy inequality to the right-hand side of (3.31), we get

$$
\left|\mathbf{v}^{T} A \mathbf{y}\right| \leq \frac{7}{6}\left(\mathbf{v}^{T} A_{0} \mathbf{v}\right)^{1 / 2}\left(\mathbf{y}^{T} A_{0} \mathbf{y}\right)^{1 / 2} .
$$

For $\mathbf{v}=\mathbf{y}$, combining this inequality with a similar inequality for the bound below, we get

$$
\frac{5}{6} \mathbf{y}^{T} A_{0} \mathbf{y} \leq \mathbf{y}^{T} A \mathbf{y} \leq \frac{7}{6} \mathbf{y}^{T} A_{0} \mathbf{y}
$$

which shows that the matrix $A$ is invertible and that the corresponding finite difference scheme has a unique solution.

Finally, let us consider the case of more accurate symmetric approximation of the fluxes at the irregular points given by (3.15). Substituting (3.15) and $\left(3.15^{\prime}\right)$ in $(3.27)$, we get

$$
\begin{aligned}
\mathbf{v}^{T} A \mathbf{y}= & \sum_{\omega}\left(\alpha^{(1)} \bar{\Delta}_{1} y \bar{\Delta}_{1} v+\alpha^{(2)} \bar{\Delta}_{2} y \bar{\Delta}_{2} v\right) \\
& +\frac{1}{4} \sum_{j=1,4,7, \ldots}\left(y_{0, j+1}-y_{0, j-1}\right)\left(v_{0, j+1}-v_{0, j-1}\right) \\
& +\frac{1}{4} \sum_{i=1,4,7, \ldots}\left(y_{i+1,0}-y_{i-1,0}\right)\left(v_{i+1,0}-v_{i-1,0}\right)
\end{aligned}
$$

where $\alpha^{(l)}=\alpha_{i, j}^{(l)}, l=1,2$, are defined by (3.29). It is obvious that the bilinear form $\mathbf{v}^{T} A \mathbf{y}$ is symmetric.

Applying the Cauchy inequality to the right-hand side of (3.34), we get

$$
\left|\mathbf{v}^{T} A \mathbf{y}\right| \leq \frac{3}{2}\left(\mathbf{v}^{T} A_{0} \mathbf{v}\right)^{1 / 2}\left(\mathbf{y}^{T} A_{0} \mathbf{y}\right)^{1 / 2} .
$$

For $\mathbf{v}=\mathbf{y}$, combining this inequality with a similar one for the bound below,

$$
\frac{1}{2} \mathbf{y}^{T} A_{0} \mathbf{y} \leq \mathbf{y}^{T} A \mathbf{y} \leq \frac{3}{2} \mathbf{y}^{T} A_{0} \mathbf{y}
$$

which shows that the matrix $A$ is invertible and that the corresponding finite difference scheme has a unique solution.

Summarizing the results, we have the following theorem for all three approximations:

Theorem 3.1. The finite difference scheme (3.16), (3.17), for which the approximate fluxes are defined by (3.5), (3.6) at the regular grid points and by any of the formulas (3.9)-(3.11) or (3.13), (3.14), or (3.15) at the irregular points, has a unique solution. Moreover, the following basic inequalities are true:

$$
\begin{gathered}
\left|\mathbf{v}^{T} A \mathbf{y}\right| \leq \gamma_{2}\left(\mathbf{v}^{T} A_{0} \mathbf{v}\right)^{1 / 2}\left(\mathbf{y}^{T} A_{0} \mathbf{y}\right)^{1 / 2}, \\
\gamma_{1} \mathbf{v}^{T} A_{0} \mathbf{v} \leq \mathbf{v}^{T} A \mathbf{v} \leq \gamma_{2} \mathbf{v}^{T} A_{0} \mathbf{v},
\end{gathered}
$$

where $A_{0}$ is the matrix of the simplest symmetric approximation, and $A$ is the matrix of any of our three approximations. If $a(x) \equiv 1$, then $\gamma_{1}=\frac{5}{6}$ and 
$\gamma_{2}=\frac{7}{6}$ for the matrix $A$ of the nonsymmetric scheme, and $\gamma_{1}=\frac{1}{2}, \gamma_{2}=\frac{3}{2}$ for the symmetric more accurate scheme.

The constants $\gamma_{1}$ and $\gamma_{2}$ in (3.37), (3.38) depend on $a(x)$. In general, this dependence is very weak. For example, if $k^{(l)}(x), l=1,2$, are evaluated exactly by (3.6), then $\gamma_{1}$ and $\gamma_{2}$ do not depend on the ratio $\max _{\Omega} a(x) / \min _{\Omega} a(x)$, which is important for the iterative methods for solving the corresponding system.

It is important to note that, in the general case $h_{c}=m h_{f}$, the derived approximations produce finite difference schemes which satisfy the inequalities (3.37) and (3.38) with constants $\gamma_{1}$ and $\gamma_{2}$ independent of the ratio $m=$ $h_{c} / h_{f}$.

\section{ERROR ESTIMATES}

In this section, we study the convergence rate of the derived finite difference schemes. As we noticed at the beginning, the cell-centered nonuniform meshes are widely used in reservoir simulation. The averaging of the coefficient using (3.6) is especially useful in the case of a piecewise continuous coefficient. The nonuniformity of the grid and the discontinuity of the coefficient raise important questions concerning convergence of the discrete solutions. Many of these questions are discussed in $[9,15-17,21]$. Since our objective is studying the problems arising from the local refinement, we concentrate on them. Thus, we consider uniform (coarse and fine) meshes and a smooth coefficient. Moreover, since the case of a smooth coefficient is similar to that of constant coefficients, we assume that $a(x) \equiv 1$.

The error analysis presented here is done in the general framework of the methods developed in [18]. First, we define the corresponding finite difference scheme for the error of the method $\varepsilon(x)=y(x)-u(x), x \in \omega$. The righthand side of the scheme is the local truncation error, which is presented in a divergence form. Next, we derive an a priori estimate for $\varepsilon(x)$ in the discrete energy norm. Finally, using the Bramble-Hilbert lemma argument [6], we get an $O\left(h^{\alpha}\right)$ convergence rate, where $\frac{1}{2} \leq \alpha$ depends on the smoothness of the exact solution $u(x)$ and on the type of the approximation we use (symmetric, nonsymmetric, or more accurate symmetric).

4.1. An a priori estimate for the error. If $\varepsilon(x)=y(x)-u(x), x \in \omega$, is the error of the finite difference method, then $y(x)=\varepsilon(x)+u(x), x \in \omega$; or, in vector form, $\mathbf{y}=\boldsymbol{\varepsilon}+\mathbf{u}$. Substituting $\mathbf{y}$ in (3.18) (or, equivalently, in (3.16), (3.17)), we obtain

$$
A \boldsymbol{\varepsilon}=\mathbf{f}-A \mathbf{u} \equiv \boldsymbol{\psi},
$$

where $A \mathrm{u}$ is defined by the left-hand side of (3.16), in which the approximate fluxes are defined by the values of $u(x)$ at the grid points. Note that $\mathbf{f}$ is defined by the right-hand sides of (3.16) and (3.17). Then, using (2.6) and the 
fact $u(x)=g(x), x \in \Gamma_{D}$, we transform $\psi$ in the form

$$
\begin{aligned}
\boldsymbol{\psi}(x) & =\sum_{l=1}^{2}\left\{\int_{s^{+}(l)} W^{(l)} d s-\stackrel{+}{w}^{(l)}-\left(\int_{s^{(l)}} W^{(l)} d s-w^{(l)}\right)\right\} \\
& \equiv \sum_{l=1}^{2}\left(\eta_{l}^{+}(x)-\eta_{l}(x)\right),
\end{aligned}
$$

where the approximate fluxes $\stackrel{+}{w}^{(l)}, w^{(l)}, l=1,2$, are defined by the values of $u(x)$ at the grid points. Here we have defined implicitly

$$
\begin{aligned}
& \eta_{l}^{+}(x)=\int_{\dot{s}^{(l)}(x)} W^{(l)} d s-\stackrel{+}{w}^{(l)}(x), \\
& \eta_{l}(x)=\int_{s^{(l)}(x)} W^{(l)} d x-w^{(l)}(x),
\end{aligned}
$$

It is easy to see that, at the regular points, $\eta_{1}^{+}\left(x_{1, i}, x_{2, j}\right)=\eta_{1}\left(x_{1, i+1}, x_{2, j}\right)$ and $\eta_{2}^{+}\left(x_{1, i}, x_{2, j}\right)=\eta_{2}\left(x_{1, i}, x_{2, j+1}\right)$. Note also that the error $\varepsilon(x)$ satisfies homogeneous Dirichlet boundary conditions on $\Gamma_{D}$; i.e.,

$$
\varepsilon(x)=0 \text { on } \Gamma_{D} \text {. }
$$

It is important to point out that $\eta_{2}^{+}(x)=0$ on the north boundary of $\Omega$, $\eta_{1}^{+}(x)=0$ on the east boundary of $\Omega$, and $\eta_{2}(x)=0$ on the south boundary of $\Omega$.

In order to investigate the convergence of the finite difference schemes, we first derive the corresponding a priori estimates for $\varepsilon(x)$ in the energy norm. We multiply equation (4.1) by $\varepsilon(x)$ and sum over all grid points $x \in \omega$. Then by (3.27) we have

$$
-\sum_{x \in \omega}\left\{w^{(1)}(x) \bar{\Delta}_{1} \varepsilon(x)+w^{(2)}(x) \bar{\Delta}_{2} \varepsilon(x)\right\}=\sum_{x \in \omega} \psi(x) \varepsilon(x) .
$$

Then, taking into account the particular form (4.2) of the local truncation error $\psi(x)$ and using the same arguments as in (3.20)-(3.27), we have

$$
\begin{aligned}
\sum_{\omega} \psi(x) \varepsilon(x) & =\sum_{\omega} \varepsilon(x) \sum_{l=1}^{2}\left(\eta_{l}^{+}(x)-\eta_{l}(x)\right) \\
& =\sum_{l=1}^{2} \sum_{\omega} \varepsilon(x)\left(\eta_{l}^{+}(x)-\eta_{l}(x)\right)=-\sum_{\omega} \sum_{l=1}^{2} \eta_{l}(x) \bar{\Delta}_{l} \varepsilon(x) .
\end{aligned}
$$

Therefore, (4.5) has the form

$$
\boldsymbol{\varepsilon}^{T} A \boldsymbol{\varepsilon} \equiv\left|\sum_{\omega} \sum_{l=1}^{2} w^{(l)} \bar{\Delta}_{l} \varepsilon\right|=\left|\sum_{\omega} \sum_{l=1}^{2} \eta_{l} \bar{\Delta}_{l} \varepsilon\right|,
$$

where the approximate fluxes $w^{(l)}, l=1,2$, are defined using the values of $\varepsilon(x)$ at the grid points $x \in \omega$. Then, applying (3.38) and estimating the righthand side by the Cauchy inequality and (3.8), we get, from (4.7),

$$
\gamma_{1} \boldsymbol{\varepsilon}^{T} A_{0} \boldsymbol{\varepsilon} \leq C\left(\boldsymbol{\varepsilon}^{T} A_{0} \boldsymbol{\varepsilon}\right)^{1 / 2}\left(\sum_{l=1}^{2} \sum_{\omega} \eta_{l}^{2}(x)\right)^{1 / 2} .
$$


Since the matrix $A_{0}$ is symmetric and positive definite (note that our vectors correspond to grid functions vanishing on $\Gamma_{D}$ ), it defines a norm, called the energy norm,

$$
\|y\|_{1, \omega} \equiv\|y\|_{A_{0}}=\left(\mathbf{y}^{T} A_{0} \mathbf{y}\right)^{1 / 2} .
$$

Using the discrete $L^{2}$-norm, defined by (2.7), (4.7), we obtain the following theorem.

Theorem 4.1. The error $\varepsilon(x)=y(x)-u(x), x \in \omega$, of the finite difference scheme (3.16), (3.17) satisfies the a priori estimate

$$
\|\varepsilon\|_{1, \omega} \leq C\left(\left\|\eta_{1}\right\|_{0, \omega}+\left\|\eta_{2}\right\|_{0, \omega}\right)
$$

where the components $\eta_{l}, l=1,2$, of the local truncation error are defined by (4.3) with approximate fluxes $\stackrel{+}{w}^{(l)}$ and $w^{(l)}, l=1,2$, determined by (3.5), (3.6), and one of the relations (3.9)-(3.11) for the simplest symmetric scheme, (3.14) for the nonsymmetric scheme, or (3.15) for the more accurate symmetric scheme. The constant $C$ does not depend on $h$ or $\varepsilon$.

Obviously, the three finite difference schemes share the same a priori estimate for the error. At this point in our development they differ only on the definition of the local truncation error $\eta_{l}, l=1,2$. In order to get an estimate for the convergence rate, we have to estimate the norms $\left\|\eta_{l}\right\|_{0, \omega}, l=1,2$, in all three cases.

4.2. Error estimates. Since we are considering the case $a(x) \equiv 1$, the components $\eta_{l}$ of the local truncation error in (4.3) are

$$
\eta_{l}(x)=\int_{s^{(l)}(x)} \frac{\partial u}{\partial x_{l}} d s-w^{(l)}(x), \quad x \in \omega,
$$

where the fluxes $w^{(l)}(x)$ are defined by the values $u(x)$ at the grid points $x \in \omega$.

Here, convergence analysis of difference schemes is performed in the framework of [18]: $\eta_{l}(x)$ are considered as linear functionals of $u(x)$, bounded in the Sobolev spaces $H^{m}, m \geq \frac{3}{2}$. For any particular finite difference approximation, these functionals vanish for polynomials of certain degree and therefore, by the Bramble-Hilbert lemma argument, are of order $O\left(h^{\alpha}\right)$ for some $\alpha$. Using these estimates in (4.10), we get the corresponding estimate for the convergence rate.

Our task now is to investigate the particular approximations of the fluxes. Let us first consider the case of regular grid points. For $l=1$, we have

$$
\eta_{1}(x)=\int_{x_{2, j}-h / 2}^{x_{2, j}+h / 2} \frac{\partial u}{\partial x_{1}}\left(x_{1, i}-\frac{1}{2} h, s\right) d s-u\left(x_{1, i}, x_{2, j}\right)+u\left(x_{1, i-1}, x_{2, j}\right) .
$$

Here, $\eta_{1}(x)$ is a linear functional of $u(x)$, bounded for $u \in H^{m}(\bar{e}), m \geq \frac{3}{2}$, $\bar{e}=\bar{e}(x)=\bar{e}_{i, j}=e_{i, j} \cup e_{i-1, j}$. This functional vanishes for all polynomials of 
second degree. Therefore, by the Bramble-Hilbert lemma argument, we get

$$
\left|\eta_{1}(x)\right| \leq C h^{m}|u|_{m+1, \bar{e}(x)}, \quad \frac{1}{2} \leq m \leq 2 .
$$

Now, let us consider the case of irregular grid points. Since at the irregular grid points we have three different approximations, we shall consider them separately.

We begin with the case of the simplest symmetric scheme, for which the approximate fluxes are defined by (3.9)-(3.11). For the points $\left(x_{1, i}, x_{2, j+l}\right)$, $l=0,1,2$, of Figure 3.2, we have

$$
\begin{aligned}
\eta_{1}\left(x_{1, i}, x_{2, j+l}\right)= & \int_{x_{2, j}+(l-1 / 2) h_{f}}^{x_{2, j}+(l+1 / 2) h_{f}} \frac{\partial u}{\partial x_{1}}\left(x_{1, i}-\frac{1}{2} h_{f}, s\right) d s \\
& -u\left(x_{1, i}, x_{2, j}+l h_{f}\right)+u\left(x_{1, i-1}, x_{2, j+1}\right) .
\end{aligned}
$$

The expression on the right is a linear functional of $u(x)$, bounded in $H^{m+1}$, $m \geq \frac{1}{2}$. For $l=0,2$, it vanishes only for $u \equiv$ const (it fails to vanish for $\left.u \equiv x_{2}\right)$. Then

$$
\left|\eta_{1}(x)\right| \leq C\left(|u|_{1, \bar{e}(x)}+h^{m}|u|_{m+1, \bar{e}(x)}\right), \quad \frac{1}{2} \leq m \leq 1, l=0,2,
$$

where $\bar{e}(x)=e_{i-1, j+1} \cup e_{i, j} \cup e_{i, j+l}, l=0,2$. The corresponding linear functional $\eta_{1}$ for $l=1$ vanishes for all polynomials of first degree, and therefore

$$
\left|\eta_{1}(x)\right| \leq C h^{m}|u|_{m+1, \bar{e}(x)}, \quad \frac{1}{2} \leq m \leq 1,
$$

with $\bar{e}(x)=e_{i-1, j+1} \cup e_{i, j}$.

If the irregular point is near the boundary $\Gamma \backslash \Gamma_{D}$, then the fluxes are defined as in the case of the simplest symmetric approximation. But since $\frac{\partial u}{\partial \nu}=0$ on $\Gamma \backslash \Gamma_{D}$, it is easy to see that the estimate (4.14) is also valid for these points.

In a similar way, we can estimate $\eta_{2}(x)$ at the other irregular points. Then, using (4.13) and (4.14), we get

$$
\begin{aligned}
\sum_{x \in \omega} \eta_{1}^{2}(x) & \leq C\left(\sum_{\substack{x \in \omega \\
x \text { irregular }}}|u|_{1, \bar{e}(x)}^{2}+\sum_{x \in \omega} h^{2 m}|u|_{m+1, e(x)}^{2}\right) \\
& \leq C\left(|u|_{1, \Omega_{h}}^{2}+h^{2 m}|u|_{m+1, \Omega}^{2}\right),
\end{aligned}
$$

where $\Omega_{h}$ is a strip of width $4 h$ around the interface between $\Omega_{1}$ and $\Omega_{2}$ (coarse and fine grid regions). The first term on the right can be estimated by the well-known $\mathrm{Il}^{\prime}$ in's inequality (see $[18$, p. 26])

$$
\|u\|_{0, \Omega_{\delta}} \leq C \delta^{1 / 2}\|u\|_{m, \Omega}, \quad m>\frac{1}{2},
$$

where $\Omega_{\delta}$ is a strip in $\Omega$ with a width $\delta$. Therefore, we have

$$
\left\|\eta_{1}\right\|_{0, \omega}=\left(\sum_{x \in \omega} \eta_{1}^{2}(x)\right)^{1 / 2} \leq C h^{1 / 2}\|u\|_{m+1, \Omega}, \quad m>\frac{1}{2}
$$


In the same way, we can estimate $\left\|\eta_{2}\right\|_{0, \omega}$. Then, by (4.10), we get:

Theorem 4.2. If the solution $u(x)$ of the problem (2.2)-(2.5) is $H^{m+1}$-regular, $m>\frac{1}{2}$, then the simplest symmetric difference scheme (3.16), (3.17), (3.5), (3.6), (3.9)-(3.11) has $O\left(h^{1 / 2}\right)$ rate of convergence in the energy norm; i.e.,

$$
\|y-u\|_{1, \omega} \leq C h^{1 / 2}\|u\|_{m+1, \Omega}, \quad m>\frac{1}{2} .
$$

The low order of convergence of the simplest symmetric difference scheme is due to its poor approximation properties at the irregular points. This is not a surprise, since this approximation was obtained by piecewise constant interpolation of the grid functions over every cell, which is a poor choice.

Now we consider the nonsymmetric approximation at the irregular point $\left(x_{1, i}, x_{2, j}\right)$ of Figure 3.2, for example:

$$
\begin{aligned}
\eta_{1}\left(x_{1, i}, x_{2, j}\right)= & \int_{x_{2, j}-h_{f} / 2}^{x_{2, j}+h_{f} / 2} \frac{\partial u}{\partial x_{1}}\left(x_{1, i}-\frac{1}{2} h_{f}, s\right) d s \\
& -\frac{1}{2}\left[u_{i, j}-\frac{2}{3} u_{i-1, j+1}-\frac{1}{3} u_{i-1, j-1}\right] .
\end{aligned}
$$

This is a linear functional of $u$, bounded in $H^{m+1}, m \geq \frac{1}{2}$, and vanishing for polynomials of first degree. Then

$$
\left|\eta_{1}(x)\right|=\left|\eta_{1}\left(x_{1, i}, x_{2, j}\right)\right| \leq C h^{m}|u|_{m+1, \bar{e}_{i, j}}, \quad \frac{1}{2} \leq m \leq 1 .
$$

Then, using the approach we described above in the case of simplest symmetric scheme, we have

$$
\left\|\eta_{1}\right\|_{0, \omega}=\left(\sum_{\omega} \eta_{1}^{2}(x)\right)^{1 / 2} \leq C h^{m+1 / 2}\|u\|_{m+3 / 2, \Omega}, \quad \frac{1}{2}<m \leq 1 .
$$

The same estimate can also be derived for the more accurate symmetric scheme, since its approximation properties are the same as those of the nonsymmetric scheme. We summarize this in the following theorem:

Theorem 4.3. If the solution $u(x)$ of the problem (2.2)-(2.5) with constant coefficient $a(x)$ is $H^{m+3 / 2}$-regular, $\frac{1}{2}<m \leq 1$, then the nonsymmetric scheme (3.16), (3.17), (3.5), (3.6), (3.14) and the more accurate symmetric scheme $(3.16),(3.17),(3.5),(3.6),(3.15)$ have $O\left(h^{m+1 / 2}\right)$ rate of convergence in the energy norm (4.9); i.e.,

$$
\|y-u\|_{1, \omega} \leq C h^{m+1 / 2}\|u\|_{m+3 / 2, \Omega}, \quad \frac{1}{2}<m \leq 1,
$$

with constant $C$ independent of $h$ and $u(x)$.

Remark 4.1. The components of the local truncation error depend locally on the solution $u(x)$. Their estimates depend only on its local smoothness. In our 
case, $\bar{\Omega}=\bar{\Omega}_{1} \cup \bar{\Omega}_{2}$, and (4.17) can be written in the form

$$
\|y-u\|_{1, \omega} \leq C\left(h_{c}^{m+1 / 2}\|u\|_{m+3 / 2, \Omega_{1}}+h_{f}^{m+1 / 2}\|u\|_{m+3 / 2, \Omega_{2}}\right) .
$$

If the term $\|u\|_{m+3 / 2, \Omega_{2}}$ is much larger than the term $\|u\|_{m+3 / 2, \Omega_{1}}$, then it is reasonable to introduce a finer grid in $\Omega_{2}$.

Unfortunately, the constant $C$ in (4.17) depends on the ratio $h_{c} / h_{f}$, and (4.18) is no longer valid for $h_{f}=h_{c}^{\beta}, \beta>1$. Instead of that, we can introduce a multilevel refinement, choosing a sequence of subdomains $\Omega_{2}^{(k+1)} \subset \Omega_{2}^{(k)}$ and introducing in each of them a mesh of size $h_{k+1}=\frac{1}{3} h_{k}, k=1,2, \ldots, l-1$. Then, in the right-hand side of (4.18) we shall have a sum of the corresponding norms of the solution in $\Omega_{2}^{(k)} \backslash \Omega_{2}^{(k+1)}$ multiplied by $h_{k}^{m+1 / 2}$, and the constant $C$ will not depend on $h$.

Numerical experiments will be reported in a forthcoming paper, which will also consider construction of optimal two-grid preconditioners for problems with local refinement.

\section{ACKNOWLEDGMENTS}

This research was supported in part by Office of Naval Research Contract No. 0014-88-K-0370, by National Science Foundation Grant No. DMS-85-4360, and by funding from the Institute for Scientific Computation at the University of Wyoming through NSF Grant No. RII-8610680. The second and the third authors have been supported also by the Bulgarian Committee of Science under Grant No. 55-26-3-87.

\section{BIBLIOGRAPHY}

1. R A. Adams, Sobolev spaces, Academic Press, New York, 1975.

2. O. Axelsson, A generalized conjugate gradient, least square method, Numer. Math. 51 (1987), 209-227.

3. O. Axelsson and V. A. Baker, Finite element solutions of boundary value problems, theory and computation, Academic Press, Orlando, 1984.

4. K. Aziz and A. Settari, Petroleum reservoir simulation, Applied Science Publishers, London, 1979.

5. J. H. Bramble, R. E. Ewing, J. E. Pasciak, and A. H. Schatz, A preconditioning technique for the efficient solution of problems with local grid refinement, Comput. Methods Appl. Mech. Engrg. 67 (1988), 149-159.

6. J. H. Bramble and S. R. Hilbert, Bounds for a class of linear functionals with application to Hermite interpolation, Numer. Math. 16 (1971), 362-369.

7. R. E. Ewing, Efficient adaptive procedures for fluid flow applications, Comput. Methods Appl. Mech. Engrg. 55 (1986), 89-103.

8. R. E. Ewing and R. D. Lazarov, Adaptive local grid refinement, Paper SPE 17806, presented at the SPE Rocky Mountain Regional Meeting, Casper, May 1988.

9. H. O. Kreiss, T. A. Manteuffel, B. Swartz, B. Wendroff, and A. B. White, Jr., Superconvergent schemes on irregular grids, Math. Comp. 47 (1986), 537-554.

10. T. A. Manteuffel and A. B. White, Jr., The numerical solution of second-order boundary value problems on nonuniform meshes, Math. Comp. 47 (1986), 511-535. 
11. S. McCormick, Fast adaptive composite grid (FAC) methods: Theory for the variational case, Comput. Suppl. 5 (1984), 115-121.

12. S. McCormick and J. Thomas, The fast adaptive composite grid (FAC) method for elliptic equations, Math. Comp. 46 (1986), 439-456.

13. O. A. Pedrosa, Jr., Use of hybrid grid in reservoir simulation, Ph. D. Thesis, Stanford University, 1984.

14. P. Quandalle and P. Besset, Reduction of grid effects due to local sub-gridding in simulations using a composite grid, Paper SPE 13527, presented at the SPE 1985 Reservoir Simulation Symposium, Dallas, February 1985.

15. A. A. Samarskii, Homogeneous difference schemes on non-uniform nets for equations of parabolic type, U.S.S.R. Comput. Math. and Math. Phys. 3 (1963), 351-393.

16. __ Introduction to the theory of difference schemes, Nauka, Moskow, 1971. (Russian)

17. __ Local one dimensional difference schemes on non-uniform nets, U.S.S.R. Comput. Math. and Math. Phys. 3 (1963), 572-619.

18. A. A. Samarskii, R. D. Lazarov, and V. L. Makarov, Difference schemes for differential equations having generalized solutions, Vysshaya Shkola, Moskow, USSR, 1987. (Russian)

19. A. N. Tikhonov and A. A. Samarskii, Homogeneous difference schemes on non-uniform nets, U.S.S.R. Comput. Math. and Math. Phys. 2 (1962), 927-953.

20. D. U. von Rosenberg, Local grid refinement for finite difference methods, Paper SPE 10974, presented at the 57th Annual Fall Technical Conference, New Orleans, September 1982.

21. A. Weiser and M. F. Wheeler, On convergence of block-centered finite differences for elliptic problems, SIAM J. Numer. Anal. 25 (1988), 351-375.

Department of Mathematics, University of Wyoming, LARAmie, Wyoming 82071

Institute of Mathematics, Bulgarian ACAdemy of Sciences, 1113 Sofia, Bulgaria

Center of Informatics and Computer Technology, Bulgarian Academy of Sciences, 1113 SOFIA, BULgaria

E-mail address: ewing\%lode@uwyo.bitnet 